\title{
The Design of The Common Urban Road Traffic Signs And Markings VR
}

\section{Cognitive Training Simulation System}

\author{
Zhenyu Li \\ Chongqing police college,Chongqing, China \\ alick320@126.com
}

Keywords: Road Traffic Signs And Markings; Cognitive Training; Simulation System; VR

\begin{abstract}
Urban road traffic signs and markings is the most effective methods and means for the implementation of road traffic control, it Is a facility for managing and controlling traffic to traffic participants by graphical symbols, colors, and text[1].Although Chinese national stands like GB5768-2009 Road traffic signs and markings, GB51038-2015 code for layout of urban road traffic signs and markings make a provision for this, Public, drivers, and even traffic police can not quickly and accurately grasp the methods to cognize the common urban road traffic signs and markings. VR is also known as the Virtual Reality technology, it can make people immersed invirtual environment which generated by the computer, direct observation of internal changes in the surrounding environment and things,give a person a kind of "feel personally on the scene."The design of the system simulates the common urban road traffic signs and markings by VR technology according to chinese relevant laws and regulations. The system can train a person how to quickly and accuratelycognize the common urban road traffic signs and markings by VR technology.
\end{abstract}

\section{Introduction}

Traffic is one of the most important standards in the world to measure the national strength level, public, drivers, and the other road traffic participants have to Improve the safety quality and safety skills - - the ability to cognize the common urban road traffic signs and markings is one of the most skills.The cognitive training methodsstill remain in the poor method like rote learning and spoon-feeding. The Ebbinghaus Forgetting Curveshows how information is lost over time when there is no attempt to retain it.The stronger the memory, the longer period of time that a person is able to recall it.VR technology can help us to achieve the target that a person can quickly and accuratelycognize the common urban road traffic signs and markings.

Virtual reality (VR) typically refers to computer technologies that use software to generate realistic images, sounds and other sensations that replicate a real environment (or create an imaginary setting), and simulate a user's physical presence in this environment, by enabling the user to interact with this space and any objects depicted therein using specialized display screens or projectors and other devices.[2]Virtual realities artificially create sensory experiences, which can include sight, touch, hearing, and, less commonly, smell. It is possible to apply VR technology to the cognitive common urban road traffic signs and markings,because of much characteristics of VR technology,just as multi sense, immersion and interactivity.The aim of the paper is to provide the references for how to design and buildthe common urban road traffic signs and markings VR cognitive training simulation system. 


\section{Introduction of development tools}

VRML(Virtual Reality Modeling Language) is a kind of modeling language used to set up the real world scene model or people's virtual 3D world. With the creation of a true 3D model, 3D virtual scene and a good interactive function. VrmlPad is developed by Graphics Parallel company, based on the text format, support for real-time preview of VRML special development tools, with VRML code download, edit, preview, debug function. Open the software, mainly divided into three major regions. Top for the common toolbar, the left side of the auxiliary area, the right side of the program debugging area. VrmlPad is a powerful and flexible VRML authoring tool, using it to design and develop VRML tools, and to create a VRML world and publish to the Internet, the supply of designers directly use.

\section{Design of system model}

The targets to develop the common urban road traffic signs and markings VR cognitive training simulation system is helping the person who participate the urban road traffic like public, drivers and traffic police to quickly and accuratelycognize the common urban road traffic signs and markings. The road traffic sign is a facility that uses graphic symbols, colors, and text to convey specific information to the traffic participants for managing traffic and ensuring safety. The road traffic marking refers to the use of lines, arrows, text, mark, and the outline of the subject in the road signs on the road surface transfer guidance, restriction, warning and other traffic information to traffic participants identified, its role is to control and guide the traffic. Any person can use the VR cognitive training simulation systeminteract with the scene, which enables participants to have a feel of the experience, through interactive way for users to experience immersion,improve the cognitive ability. The system consists of VR static simulation module,VR dynamicsimulation module,GUI and auxiliary training system, the website publishing.

The common urban road traffic signs and markings VR cognitive training simulation system

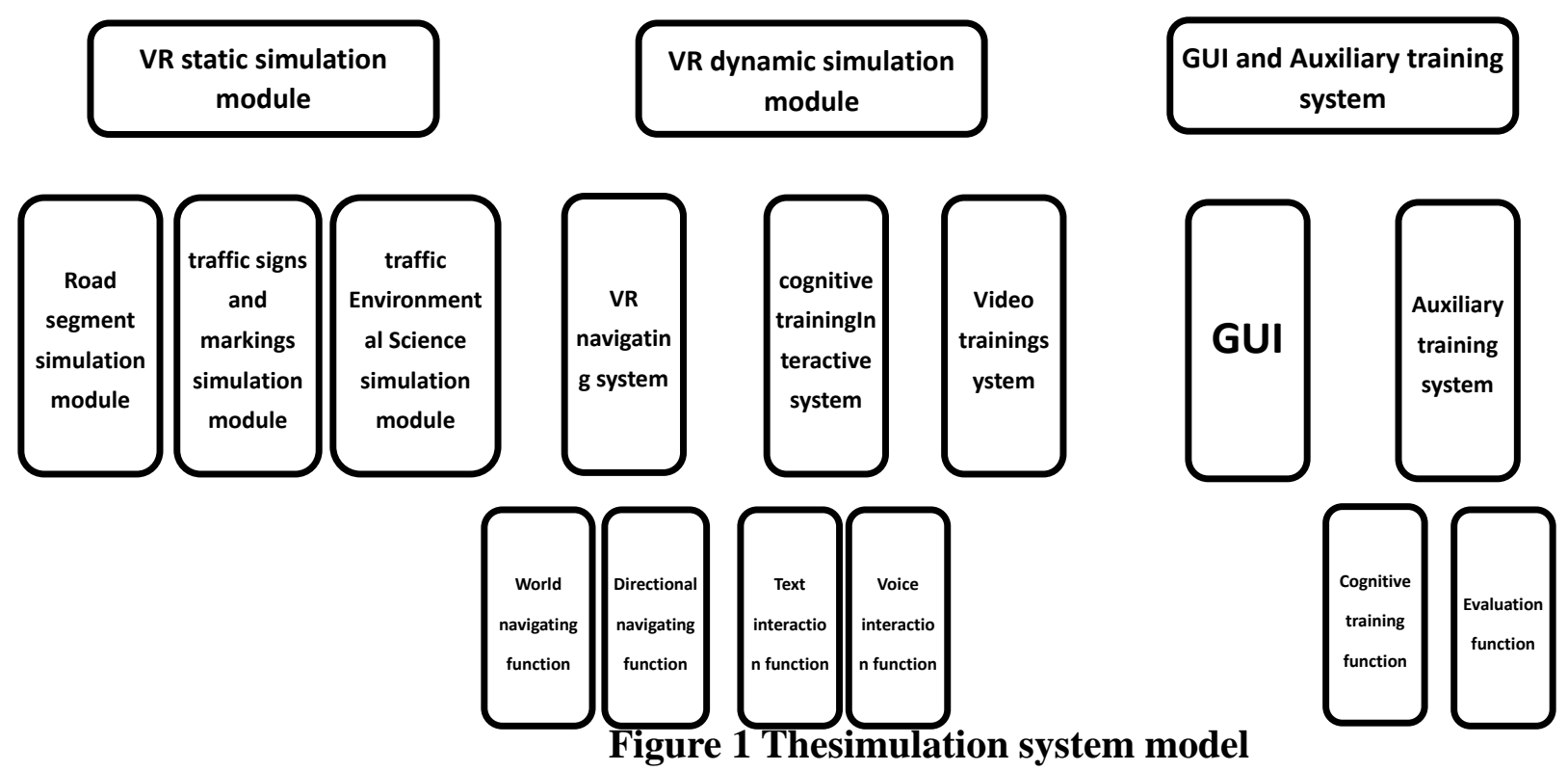

VR static simulation module.VR static simulation module means the simulation facility that does not change with time and is relatively static.It consists of normal road segment and intersectionsimulation module, traffic signs and markings simulation module, traffic Environmental 
Science simulation module.The system consists of9 cross-intersections, 4 T-intersections, 10 two-lane segments, 4 single-lane segments,18 kinds of the road traffic signs which including mandatory signs,prohibition signs,warning signs,trunk and branch road guide signs,urban expressway guide signs, 15 kinds of the road trafficmarkings which including mandatory markings,prohibition markings, warningmarkings.

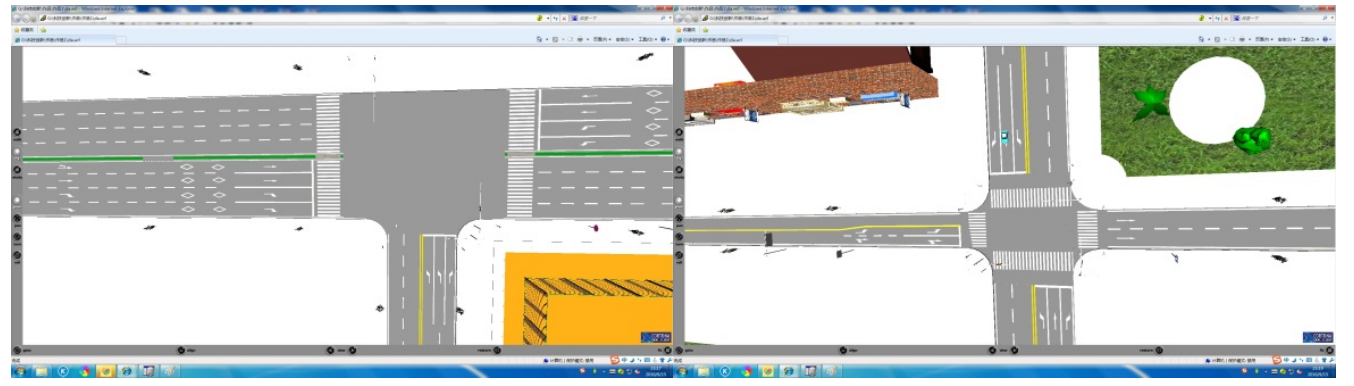

Figure2 Aerial view of the urbanroad segment and intersection simulation module

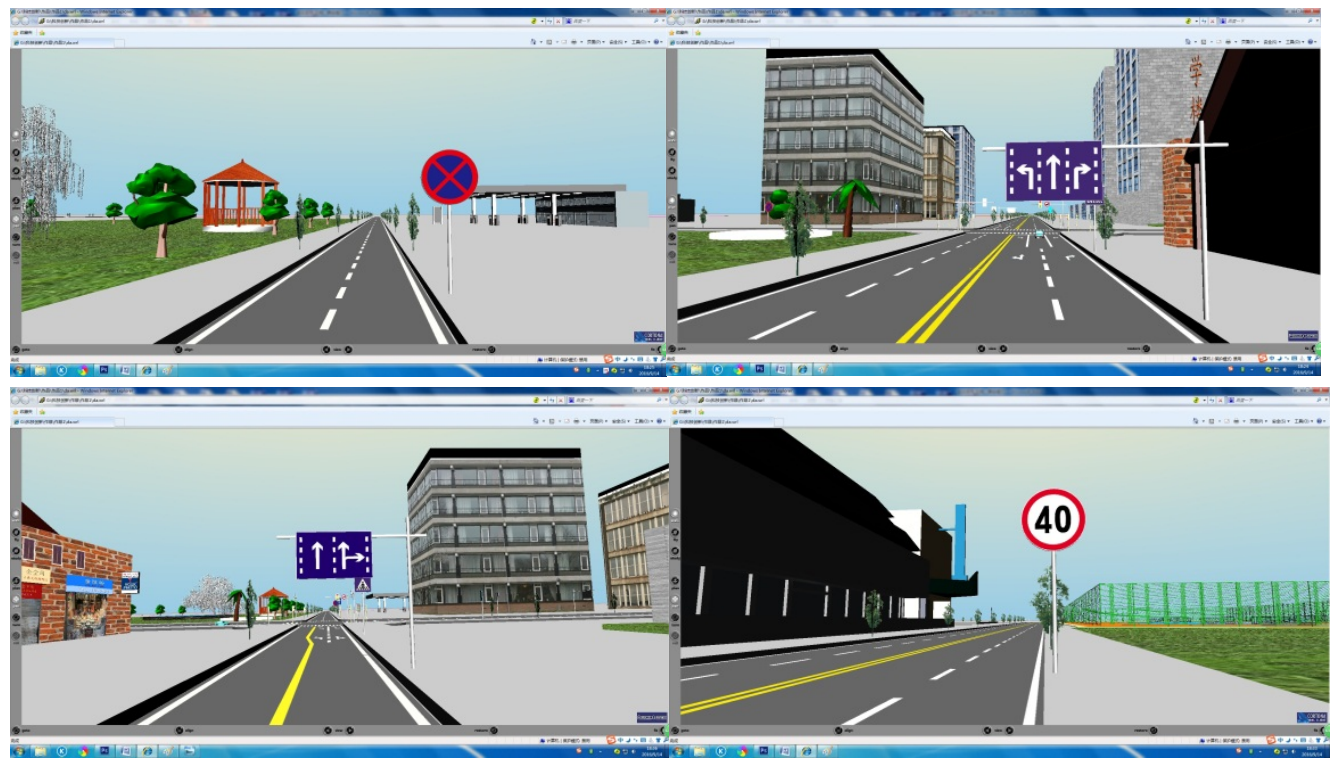

Figure3 View of the normal road traffic signs in the simulation system

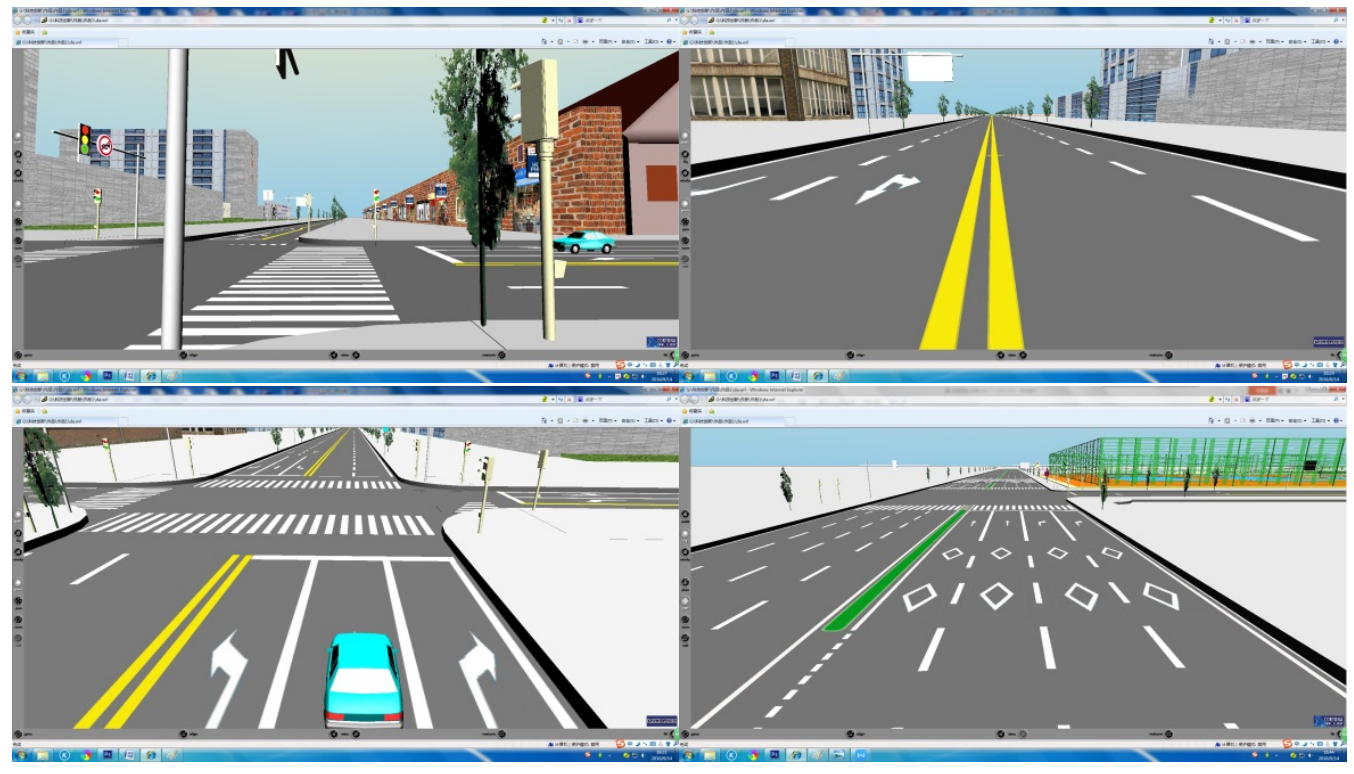

Figure4 View of the normal road traffic markings in the simulation system 
VR dynamic simulation module. Users can use the human-computer interaction, text and voice prompts, video teaching and training to achieve the goal that any userquickly and accuratelycognize the common urban road traffic signs and markings. VRML supports a variety of scripting languages,including VRMLScript,JavaScript,JAVA.m

JavaScript is the safe script language which bases on object.Unlike the Java language, JavaScript language is a basic feature of the object based on the event driven, the graphical interface environment, complete control of the virtual reality of the static objects, in order to achieve text conversion, voice conversion, video playback of the virtual reality scene dynamic function.Because of the advantages of simplicity, security, dynamic, cross platform, JavaScript language is used to developVR dynamic simulation module of the system. VR dynamic simulation module of the simulation system consists of VR navigating system, cognitive training Interactive system, Video training system.

VR navigating system includes world navigating function and directional navigating function.Users can navigate in the VR system from all aspects in the virtual wordby the First-person perspective under the world navigating model,and also navigate in the scene which designate the road segment and intersection to learn theappropriatetraffic signs and markings.

Cognitive training Interactive system includes text interaction function and voice interaction function.Users can strengthen the understanding of the graphical symbols and the environment, and then strengthen the semantic understanding, so as to strengthen the ability of cognition and recognition by text and voice interaction prompt function. So that's the basic flow:

First, when the user is in a state of visual navigating, no text and voice prompts appear;

Then, when the user moves the mouse or the data glove object needs to shift in the cognition, the mouse display indicator for finger state, meaning can display text;

Third, when the user clicks the mouse or the data glove, the simulation system will display the related text prompt board in the object side;

Finally, when the user clicks the mouse to another target, the text prompts the board to disappear.

\section{Conclusion}

This system has the advantages of convenient operation, intuitive, strong learning and sharing strong advantages,thanks to the VRML language and JAVA language and IE object browser compatible, the system can be published on the Internet, anyone can access through the IE browser, and the realization of interactive cognitive simulation training function.

\section{Acknowledgement}

Fund Project: This paper is supported by Foundation of 2016 Science and technology project of Chongqing Municipal Education Commission,Project number:KJ1601505.

\section{References}

[1]Xu Xiao-hui and Tang Hong, Road traffic management[M].People's Public Security University of China Press, 2014.

[2]Xue Qing-wen and Xin Yun-dong,Virtual reality VRML program design and examples[M].Tsinghua University Press,2012. 